\title{
CONVERGENCE ANALYSIS OF AN EXPLICIT SPLITTING METHOD FOR LASER PLASMA INTERACTION SIMULATIONS*
}

\author{
GEORG JANSING $^{\dagger}$ AND ACHIM SCHÄDLE ${ }^{\dagger}$
}

\begin{abstract}
The convergence of a triple splitting method originally proposed by Tückmantel, Pukhov, Liljo, and Hochbruck for the solution of a simple model that describes laser plasma interactions with overdense plasmas is analyzed. For classical explicit integrators it is the large density parameter that imposes a restriction on the time step size to make the integration stable. The triple splitting method contains an exponential integrator in its central component and was specifically designed for systems that describe laser plasma interactions and overcomes this restriction. We rigorously analyze a slightly generalized version of the original method. This analysis enables us to identify modifications of the original scheme such that a second-order convergent scheme is obtained.
\end{abstract}

Key words. exponential integrators, highly oscillatory problems, trigonometric integrators, splitting methods

AMS subject classifications. 65P10

1. Introduction. We consider the numerical solution of a system of equations describing laser plasma interactions with an overdense plasma that is a simplified version of the models considered in [14, 16]. Essentially as in [17], the laser is described by Maxwell's equations, and the plasma is modeled as a fluid only, in contrast to $[14,16]$. After discretizing in space with a fixed spatial grid size $h$, a system of ordinary differential equations is obtained. This highly oscillatory system of ordinary differential equations is discretized in time by a triple splitting method with filter functions. The introduction of filter functions is a widely-used method to avoid resonance effects in splitting methods applied to oscillatory differential equations; see, e.g., $[4,6,7,11]$ and [8].

The situation to consider now is slightly unusual as it is the localized overdense plasma and not the spatial discretization that gives rise to fast oscillations in the solution. The plasma frequency is several orders of magnitude larger than the laser frequency, which has to be resolved by the spatial grid. Hence, it is the plasma density $\rho$ that imposes a step size restriction in explicit Runge-Kutta or multistep methods. To overcome the restriction on the time step size due to the plasma density, a triple splitting method with filter functions was introduced in $[13,17]$ for this model problem. An astute choice of filter functions results in a method that shows excellent behavior in numerical experiments. The numerical experiments in [17] indicate convergence of second order in the time step size $\tau$ independent of $\rho$. A more detailed experiment, which is reported in Section 8.1, reveals that the method from [17] is not of second order in $\tau$ independent of the plasma density but is merely stable.

By our convergence analysis of the triple splitting we are able to formulate conditions on the filter functions to obtain second-order convergence in $\tau$ independent of the plasma density $\rho$. These conditions can be fulfilled by slightly modifying the choice of the filter functions originally proposed in $[13,17]$. The modification comes at no additional computational cost.

As the triple splitting is an explicit integrator, the method certainly can not be expected to be convergent uniformly as $h \rightarrow 0$, where $h$ is the spatial discretization parameter. In regions without plasma, our method reduces to the leapfrog method such that the usual CFL condition, depending on the spatial discretization, is certainly necessary for stability. Our aim here is to prove convergence independent of the large plasma density $\rho$ but not independent of $h$.

* Received August 25, 2017. Accepted April 24, 2018. Published online on June 28, 2018. Recommended by Marlis Hochbruck.

${ }^{\dagger}$ Mathematisches Insititut, Heinrich-Heine Universität, Universitätsstraße 1, 40225 Düsseldorf, Germany ( $\{$ jansing, schaedle\}@hhu.de). 
In a nutshell, the idea for the convergence proof is as follows: The triple splitting for the impulse of the plasma $\mathbf{p}$, the electric field $\mathbf{e}$, and the magnetic flux $\mathbf{b}$ will be reformulated as a two-step method for e only with some sort of "natural" filters. Perturbing the initial values, this reformulation allows us to estimate the error in e using [8, Theorem XIII.4.1]. We then show that the perturbation in the initial values is small enough such that by a stability argument, convergence for $\mathbf{e}$ is obtained. The estimates for the magnetic flux $\mathbf{b}$ and the impulse $\mathbf{p}$ are obtained by a judicious combination of ideas borrowed from [6] with trigonometric identities. The present paper is based on the first part of [12].

2. The physical problem and its spatial discretization. Consider the propagation of a short laser pulse in vacuum targeted at a plasma around a thin foil. The electric field e and the magnetic flux $\mathbf{b}$ describing the laser are governed by Maxwell's equations. In this simple model the plasma is modeled as a fluid by the electron number density $\rho$ (number of electrons per volume) and the probability density function $\mathbf{p}$ such that $\rho \mathbf{p}$ is the impulse of the electrons. We will use a somewhat sloppy notation and let $\mathbf{p}$ denote an impulse in what follows. The laser plasma interactions with an overdense plasma $(\rho \gg 1)$ and a linear response of the plasma to the laser are modeled by

$$
\begin{array}{ll}
\partial_{t} \mathbf{p}=\mathbf{e}, & x \in B, t>0, \\
\partial_{t} \mathbf{e}=\nabla \times \mathbf{b}-f^{2} \rho \mathbf{p}, & x \in B, t>0, \\
\partial_{t} \mathbf{b}=-\nabla \times \mathbf{e}, & x \in B, t>0 .
\end{array}
$$

Here $f=2 \pi e$, where $e$, the electron charge, is a constant. $B$ is the computational domain, a box containing the plasma and the support of the initial values. The vacuum permittivity (electric constant) and permeability (magnetic constant) are set to 1 . In our simplified model, plasma only oscillates locally, thus its impulse $\mathbf{p}$ also oscillates, but the density $\rho$ remains constant. There are two further essential assumptions. We assume that the electrons move slowly such that relativistic effects can be neglected, i.e., the velocity field of the plasma $\mathbf{v}$ is proportional to the impulse $\mathbf{p}$. Secondly, we neglect the magnetic Lorentz force $\mathbf{v} \times \mathbf{b}$. These rather restrictive assumptions make the model (2.1) linear. A more detailed derivation of the model may be found in $[15,17]$.

Equation (2.1) has to be supplemented with boundary conditions and initial values. The theory developed below applies to the case of a perfect magnetic conductor (PMC), a perfect electric conductor (PEC), or periodic boundary conditions, which guarantee that the "curl curl" operator is self-adjoint; see [10].

As we only discuss the convergence of the semi-discrete problem in the following, the solution of the spatially discretized equations will again be denoted by $\mathbf{p}, \mathbf{e}$, and $\mathbf{b}$. Discretizing in space with the Yee scheme or curl-conforming finite elements, we denote by $\mathbf{C}_{E}$ and $\mathbf{C}_{B}$ the discrete versions of the "curl" applied to $\mathbf{e}$ and $\mathbf{b}$, respectively. The electric field e can conveniently be interpreted as a differential 1-form, and then $\mathbf{C}_{E}$ is a discrete version of "curl". Whereas in this context, $\mathbf{b}$ has to be interpreted as differential 2 -form such that $\mathbf{C}_{B}$ is as discrete version of "*curl*", where * is the Hodge operator; see [9]. Hence, the two $\nabla \times$-operators acting on either $\mathbf{e}$ or $\mathbf{b}$ are different.

The multiplication with $f^{2} \rho$ is discretized by a matrix $\Omega^{2}$. In case of the Yee scheme, $\Omega^{2}$ is a diagonal matrix. In case one uses curl-conforming finite elements, $\Omega^{2}$ is a positive semi-definite matrix, and mass matrices arise on the right-hand side of (2.1). In what follows we assume that $\Omega^{2}$ is a diagonal matrix with only one positive eigenvalue. Generalizations to a non-diagonal but symmetric positive semi-definite discretization $\Omega^{2}$ of the multiplication operator will be discussed in Section 7. 
If space is scaled with respect to the wave number and time with respect to the laser frequency, then the spatially discretized equations are

$$
\begin{array}{rlrl}
\partial_{t} \mathbf{p} & =\mathbf{e}, & t>0 \\
\partial_{t} \mathbf{e}=\mathbf{C}_{B} \mathbf{b}-\mathbf{\Omega}^{2} \mathbf{p}, & t>0 \\
\partial_{t} \mathbf{b}=-\mathbf{C}_{E} \mathbf{e}, & t>0
\end{array}
$$

Assuming for the moment that $\rho$ vanishes, a right traveling pulse with width-parameter $\sigma_{0}$ and wavelength 1 solving (2.1) is given by

$$
\begin{aligned}
& \mathbf{e}_{y}=\mathbf{b}_{z}=a_{0} \exp \left(-\frac{(2 \pi[(x-\bar{x})-t])^{2}}{2 \sigma_{0}^{2}}\right) \cos (2 \pi[(x-\bar{x})-t]), \\
& \mathbf{e}_{x} \equiv \mathbf{e}_{z} \equiv \mathbf{b}_{x} \equiv \mathbf{b}_{y} \equiv 0 .
\end{aligned}
$$

In Figure 2.1, snapshots of the solution at different times from an exemplary simulation are shown to illustrate the interaction of the pulse with an overdense plasma, i.e., $\rho>1$. There, the spatially one-dimensional case with $B=[0,30]$ is considered. Setting $t=0, \bar{x}=10$, $\sigma_{0}=10$, and $a_{0}=1$ in (2.3), the initial values for the fields and the impulse were chosen leading to a pulse centered at 10 with amplitude 1 traveling in positive $x$-direction. The plasma is situated away from the initial location of the pulse by taking $\rho=10^{8}$ for $x \in[20,21]$ and $\rho=0$ elsewhere. This leads to a total reflection of the laser pulse on the edge of the plasma. It looks odd that parts of $\mathbf{p}$ remain around $\bar{x}$. However, as the density $\rho$ is zero there, so is the impulse $\rho \mathbf{p}$ such that this is not a nonphysical behavior. The spatial derivatives are approximated via second-order central finite differences on a staggered grid, where we use $N+1=241$ grid points for $\mathbf{e}, \mathbf{b}$, and $\mathbf{p}$ each. These spatial discretization parameters are also used in the numerical experiments in Section 8.1.


FIG. 2.1. Solution of (2.1) with the laser pulse from (2.3) as initial value computed using the matrix exponential of the discretized operators. Impulse $\mathbf{p}_{y}$ (green), electric field $\mathbf{e}_{y}$ (red), magnetic flux $\mathbf{b}_{z}$ (blue), and electron density $\rho$ (magenta) scaled to one. First plot $(t=0)$ : initial data; second $(t=5)$ : propagation of the pulse in vacuum; third $(t=10)$ : total reflection at the foil; fourth $(t=20)$ : back propagation. 
3. Numerical scheme and filter functions. To solve the spatially discretized equations (2.2), we use the triple splitting method proposed in [13,17]. To this end the right-hand side is split into three terms

$$
\partial_{t}\left[\begin{array}{l}
\mathbf{p} \\
\mathbf{e} \\
\mathbf{b}
\end{array}\right]=\left[\begin{array}{c}
0 \\
0 \\
-\mathbf{C}_{E} \mathbf{e}
\end{array}\right]+\left[\begin{array}{c}
0 \\
\mathbf{C}_{B} \mathbf{b} \\
0
\end{array}\right]+\left[\begin{array}{ccc}
0 & 1 & 0 \\
-\boldsymbol{\Omega}^{2} & 0 & 0 \\
0 & 0 & 0
\end{array}\right]\left[\begin{array}{l}
\mathbf{p} \\
\mathbf{e} \\
\mathbf{b}
\end{array}\right]:=f_{1}+f_{2}+f_{3}
$$

The fully discrete scheme is a symmetric triple splitting obtained by taking the exact flows of the split equations (i.e., with only one $f_{i}$ as right-hand side) as propagators. As already observed in $[13,17]$, due to resonances, this is not sufficient for convergence independent of $\rho$. We follow $[13,17]$, introduce filter functions symmetrically and obtain the following numerical scheme:

$$
\begin{aligned}
\mathbf{b}_{n+\frac{1}{2}} & =\mathbf{b}_{n}-\frac{\tau}{2} \psi_{B}\left(\frac{\tau}{2} \boldsymbol{\Omega}\right) \mathbf{C}_{E} \phi_{E}\left(\frac{\tau}{2} \boldsymbol{\Omega}\right) \mathbf{e}_{n}, \\
\mathbf{e}_{n}^{+} & =\mathbf{e}_{n}+\frac{\tau}{2} \psi_{E}\left(\frac{\tau}{2} \boldsymbol{\Omega}\right) \mathbf{C}_{B} \phi_{B}\left(\frac{\tau}{2} \boldsymbol{\Omega}\right) \mathbf{b}_{n+\frac{1}{2}}, \\
{\left[\begin{array}{c}
\mathbf{p}_{n+1} \\
\mathbf{e}_{n+1}^{-}
\end{array}\right] } & =\left[\begin{array}{cc}
\cos (\tau \boldsymbol{\Omega}) & \tau \operatorname{sinc}(\tau \boldsymbol{\Omega}) \\
-\boldsymbol{\Omega} \sin (\tau \boldsymbol{\Omega}) & \cos (\tau \boldsymbol{\Omega})
\end{array}\right]\left[\begin{array}{c}
\mathbf{p}_{n} \\
\mathbf{e}_{n}^{+}
\end{array}\right], \\
\mathbf{e}_{n+1} & =\mathbf{e}_{n+1}^{-}+\frac{\tau}{2} \psi_{E}\left(\frac{\tau}{2} \boldsymbol{\Omega}\right) \mathbf{C}_{B} \phi_{B}\left(\frac{\tau}{2} \boldsymbol{\Omega}\right) \mathbf{b}_{n+\frac{1}{2}}, \\
\mathbf{b}_{n+1} & =\mathbf{b}_{n+\frac{1}{2}}-\frac{\tau}{2} \psi_{B}\left(\frac{\tau}{2} \boldsymbol{\Omega}\right) \mathbf{C}_{E} \phi_{E}\left(\frac{\tau}{2} \boldsymbol{\Omega}\right) \mathbf{e}_{n+1} .
\end{aligned}
$$

For $i \in\{E, B\}$, we require $\psi_{i}, \phi_{i}$ to be even, analytic functions such that $\psi_{i}(z), \phi_{i}(z) \rightarrow 1$ for $z \rightarrow 0$.

In the following section we state assumptions on the physical data and the spatial discretization that are necessary for the convergence proof.

4. Assumptions. The following assumptions are not too restrictive from a theoretical physics point of view. They are fulfilled for example in the situation considered in [13, 17] simulating the reflection of a laser pulse by a plasma.

ASSUMPTION 4.1. We assume that

(i) the product $\mathbf{C}_{B} \mathbf{C}_{E}=:-\mathbf{G}=-\mathbf{G}^{T}$ is symmetric, positive semi-definite,

(ii) $\boldsymbol{\Omega}$ is a diagonal matrix given by

$$
\boldsymbol{\Omega}=\left[\begin{array}{cc}
0 & 0 \\
0 & \widetilde{\omega} \mathrm{Id}
\end{array}\right], \quad \widetilde{\omega} \gg 1, \quad \text { and }
$$

$$
\left\|\mathbf{C}_{E}\right\| \leq C_{c} \quad \text { and } \quad\left\|\mathbf{C}_{B}\right\| \leq C_{c} \quad \text { such that } \quad\|\mathbf{G}\| \leq C_{g}:=C_{c}^{2},
$$

with a constant $C_{c}$ independent of $\widetilde{\omega}$.

The symmetry and negative semi-definiteness of $\mathbf{G}$ comes quite natural provided that the continuous "curl curl" operator is self-adjoint and positive semi-definite, which is the case for appropriate boundary conditions such as a perfect electric conductor (PEC), a perfect magnetic conductor (PMC), or periodic boundary conditions [10]. Condition (4.1) implies that the matrix $\Omega$ has only one (large) non-zero eigenvalue $\widetilde{\omega}>0$. In our case it is given by the density parameter, i.e., $\widetilde{\omega}=f \sqrt{\rho}$. This is an essential restriction, which is only needed in the proof of Theorem 6.5. A modification of our proof that only requires $\Omega$ to be symmetric positive semi-definite is given in [2] and is discussed in Section 7. Here $\Omega$ is a diagonal matrix and the filter functions are evaluated once on the diagonal elements in the beginning, and the application of the filter function reduces to an elementwise product of two vectors. If $\boldsymbol{\Omega}$ is not a diagonal matrix, then the evaluation of the action of the filter functions applied to a vector 
can become prohibitively expensive. The estimates (4.2) imply that we do not obtain error bounds uniformly in the spatial discretization parameter, e.g., the mesh width. As mentioned already in the introduction, this would indeed be impossible for our integration scheme since it reduces to the Störmer-Verlet method in case of no material $(\rho \equiv 0)$, which is known to be conditionally stable only. Assumption 4.1 is for example satisfied if the curl operators with periodic boundary conditions are discretized using a Yee-scheme and a step function $\rho$ is evaluated pointwise.

Additionally we need some bounds on the initial data $\mathbf{e}_{0}, \mathbf{b}_{0}$, and $\mathbf{p}_{0}$ to obtain stable solutions as discussed in Section 6.1:

ASSUMPTION 4.2. We assume that

$$
\begin{aligned}
\left\|\mathbf{\Omega}_{0}\right\|^{2} & \leq \frac{2}{3} H_{0}, \quad\left\|\mathbf{C}_{B} \mathbf{b}_{0}\right\|^{2} \leq \frac{1}{3} \min \left\{1, \frac{4}{C_{4}^{2}}\right\} H_{0}, \quad\left\|\boldsymbol{\Omega}^{2} \mathbf{p}_{0}\right\|^{2} \leq \frac{1}{3} H_{0}, \\
-\left\langle\mathbf{e}_{0}, \mathbf{G e}_{0}\right\rangle & =\left\|\mathbf{C}_{E} \mathbf{e}_{0}\right\|^{2} \leq 2 H_{0}, \quad\left\|\mathbf{e}_{0}\right\|^{2} \leq H_{0}, \\
\left\|\mathbf{b}_{0}\right\|^{2} & \leq H_{0} \quad \text { and }\left\|\boldsymbol{\Omega}^{2} \mathbf{C}_{B} \mathbf{b}_{0}\right\|^{2} \leq H_{0},
\end{aligned}
$$

with constants $H_{0}$ and $C_{4}$ independent of $\widetilde{\omega} . C_{4}$ is the constant given in (5.2d) below.

A bound with respect to multiplication with $\Omega$ implies that the initial data are sufficiently far away from the plasma such that the product of the field strength and the density is bounded independent of the density. Bounds with respect to multiplications with $\mathbf{C}_{B}$ or $\mathbf{C}_{E}$ may be seen as smoothness conditions for the initial data.

5. Main theorem. For our convergence result we need conditions on the filter functions, which we collect below. As in [17] we require

$$
\phi_{B} \equiv \psi_{B} \equiv 1 .
$$

The following bounds for the filter functions, which hold, for example, for $\psi_{E}(z)=\operatorname{sinc}(z)$ and $\phi_{E}(z)=\operatorname{sinc}^{2}(z)$, are required for second-order convergence of the scheme (3.1):

$$
\begin{aligned}
\left|(\cos (z)+1) \psi_{E}\left(\frac{1}{2} z\right)\right| & \leq C_{1} \operatorname{sinc}^{2}\left(\frac{1}{2} z\right), \\
\left|\phi_{E}\left(\frac{1}{2} z\right)\right| & \leq C_{2}\left|\operatorname{sinc}\left(\frac{1}{2} z\right)\right|, \\
\left|(\cos (z)+1) \psi_{E}\left(\frac{1}{2} z\right) \phi_{E}\left(\frac{1}{2} z\right)\right| & \leq C_{3}|\operatorname{sinc}(z)|, \\
\left|(\cos (z)+1) \psi_{E}\left(\frac{1}{2} z\right)\right| & \leq C_{4}|\operatorname{sinc}(z)|, \\
\left|\operatorname{sinc}(z)-\frac{1}{2}(\cos (z)+1) \psi_{E}\left(\frac{1}{2} z\right)\right| & \leq C_{5} z^{2}|\operatorname{sinc}(z)|, \\
\left|\operatorname{sinc}(z)-\phi_{E}\left(\frac{1}{2} z\right)\right| & \leq C_{6}\left|z \sin \left(\frac{1}{2} z\right)\right|, \\
\left|\psi_{E}(z)\right| & \leq C_{7}, \\
\left|\operatorname{sinc}^{2}\left(\frac{1}{2} z\right)-\operatorname{sinc}(z) \phi_{E}\left(\frac{1}{2} z\right)\right| & \leq C_{8} \sin ^{2}\left(\frac{1}{2} z\right) .
\end{aligned}
$$

With these conditions we obtain our main result

TheORem 5.1. Let $\mathbf{C}_{B}, \mathbf{C}_{E}$, and $\boldsymbol{\Omega}$ be such that Assumption 4.1 is fulfilled. Consider the numerical solution of the system (2.2) by the splitting method (3.1) with a time step size $\tau$ satisfying $\tau \leq \tau_{0}$ for sufficiently small $\tau_{0}$ independent of $\widetilde{\omega}$ such that $\tau \widetilde{\omega} \geq c_{0}>0$ for some constant $c_{0}$. If the initial values satisfy conditions (4.3)-(4.5) with a constant $H_{0}$ independent of $\widetilde{\omega}$ and the filter functions satisfy (5.2a)-(5.2h), then for $t_{n}:=t_{0}+n \tau \leq T$, we obtain the following second-order estimates for the errors

$$
\left\|\mathbf{p}_{n}-\mathbf{p}\left(t_{n}\right)\right\| \leq C \tau^{2}, \quad\left\|\mathbf{e}_{n}-\mathbf{e}\left(t_{n}\right)\right\| \leq C \tau^{2}, \quad\left\|\mathbf{b}_{n}-\mathbf{b}\left(t_{n}\right)\right\| \leq C \tau^{2} .
$$

The constant $C$ is independent of $\widetilde{\omega}, \tau, n$, and the derivatives of the solution, but it depends on $\left(T-t_{0}\right)$, the constants $c_{0}, C_{1}, \ldots, C_{8}$ in (5.2), $C_{c}$ in (4.2), and $H_{0}$. 
The assumption $\tau \widetilde{\omega} \geq c_{0}>0$ comprise the interesting case and is no restriction as otherwise we are in the case of the classical convergence analysis. The proof is given in Section 6.

Choice of the filter functions. In [17] the authors propose the choice

$$
\phi_{E}=\psi_{E}=z \mapsto \operatorname{sinc}(z), \quad \phi_{B} \equiv \psi_{B} \equiv 1
$$

which satisfies the conditions $(5.2 \mathrm{~b})-(5.2 \mathrm{~h})$. It does not obey condition (5.2a) but only the weaker estimate $\left|(\cos (z)+1) \psi_{E}\left(\frac{1}{2} z\right)\right| \leq C_{0} \operatorname{sinc}\left(\frac{1}{2} z\right)$. Detailed numerical tests in Section 8.1 for this choice reveal sharp resonances at even multiples of $\pi / \widetilde{\omega}$, which where not observed in [17], presumably due to an underresolved numerical experiment.

We propose the new choice

$$
\phi_{E}=z \mapsto \operatorname{sinc}(z), \quad \psi_{E}=z \mapsto \operatorname{sinc}^{2}(z), \quad \phi_{B} \equiv \psi_{B} \equiv 1,
$$

which also satisfies the first filter condition (5.2a) and thus by Theorem 5.1 results in secondorder error bounds. The detailed proof that the filter functions (5.4) meet all the conditions (5.2) can be found in [12, Section 4.12].

These two choices of filter functions are used in the numerical experiment in Section 8.1. Figure 8.1 there displays the error for the scheme (3.1) without filter ("No Filter"), with the filter choice (5.4) ("New"), which yields a second-order scheme uniformly in $\widetilde{\omega}$, and with the filter choice (5.3) ("Orig"), which violates (5.2a) and shows sharp resonances and a breakdown of the method if $\tau \widetilde{\omega}$ is close to even multiples of $\pi$.

REMARK 5.2. Theorem 4.19 in [12] claims that for the filter choice (5.3) one obtains convergence of order one in $\tau$ independent of $\omega$. The numerical experiment in Section 8.1 is a counterexample to this claim.

Theorem 4.19 of [12] is based on the first-order convergence result for the two-step method (6.7) given in [8, Theorem XIII.4.1] for the weakened filter assumption $\left|(\cos (z)+1) \psi_{E}\left(\frac{1}{2} z\right)\right| \leq C_{0} \operatorname{sinc}\left(\frac{1}{2} z\right)$ replacing $(5.2 \mathrm{a})$. In Section 8.2 we give a counterexample to this first-order convergence result; see also Remark 6.2.

6. Proof of Theorem 5.1. The proof is divided into four steps. First we reformulate the scheme (3.1) as a two-step method for the electric field e only. With this reformulation we can apply an already known error estimate to control the error in the electric field after modifying the initial values. Based on the error bound for $\mathbf{e}$, error bounds for $\mathbf{b}$ and $\mathbf{p}$ are obtained. A more detailed proof can be found in [12, Chapter 4].

6.1. Reformulation. From equation (2.2) one obtains an equation for the electric field

$$
\left\{\begin{array}{l}
\partial_{t t} \mathbf{e}(t)=-\boldsymbol{\Omega}^{2} \mathbf{e}(t)+\mathbf{G e}(t), \\
\mathbf{e}\left(t_{0}\right)=\mathbf{e}_{0}, \quad \partial_{t} \mathbf{e}\left(t_{0}\right)=\mathbf{C}_{B} \mathbf{b}\left(t_{0}\right)-\boldsymbol{\Omega}^{2} \mathbf{p}\left(t_{0}\right):=\dot{\mathbf{e}}_{0}
\end{array}\right.
$$

with the Hamiltonian

$$
\mathcal{H}(\mathbf{e}, \mathbf{f})=\frac{1}{2}\|\mathbf{f}\|^{2}+\frac{1}{2}\|\mathbf{\Omega} \mathbf{e}\|^{2}-\frac{1}{2}\langle\mathbf{e}, \mathbf{G e}\rangle=\frac{1}{2}\|\mathbf{f}\|^{2}+\frac{1}{2}\|\boldsymbol{\Omega} \mathbf{e}\|^{2}+\frac{1}{2}\left\|\mathbf{C}_{E} \mathbf{e}\right\|^{2},
$$

where $\mathbf{f}=\partial_{t} \mathbf{e}$. From Assumption 4.2 we deduce the stability estimates

$$
\begin{aligned}
\mathcal{H}\left(\mathbf{e}(t), \partial_{t} \mathbf{e}(t)\right) & \leq 2 H_{0}, \\
\|\mathbf{e}(t)\| & \leq\left(1+2\left(T-t_{0}\right)\right) \sqrt{H_{0}}, \\
\|\mathbf{b}(t)\| & \leq\left(1+2\left(T-t_{0}\right)\right) \sqrt{H_{0}}
\end{aligned}
$$


for $t_{0} \leq t \leq T$. The latter two can be obtained by expressing $\mathbf{e}(t)$ and $\mathbf{b}(t)$ through the fundamental theorem of calculus and exploiting that the integrands $\partial_{t} \mathbf{e}$ and $-\mathbf{C}_{E} \mathbf{e}$ are both bounded by the Hamiltonian. The formula for the variation of constants gives the following representation of the solution $\mathbf{e}$ of (6.1) starting from $t^{\prime}$ with initial data $\mathbf{e}\left(t^{\prime}\right)$ and $\partial_{t} \mathbf{e}\left(t^{\prime}\right)$ :

$$
\begin{aligned}
\mathbf{e}(t)= & \cos \left(\left(t-t^{\prime}\right) \boldsymbol{\Omega}\right) \mathbf{e}\left(t^{\prime}\right)+\left(t-t^{\prime}\right) \operatorname{sinc}\left(\left(t-t^{\prime}\right) \boldsymbol{\Omega}\right) \partial_{t} \mathbf{e}\left(t^{\prime}\right) \\
& +\left(t-t^{\prime}\right) \int_{0}^{1}\left(t-t^{\prime}\right)(1-\xi) \operatorname{sinc}\left(\left(t-t^{\prime}\right)(1-\xi) \boldsymbol{\Omega}\right) \mathbf{G e}\left(t^{\prime}(1-\xi)+t \xi\right) \mathrm{d} \xi
\end{aligned}
$$

and similarly for $\partial_{t} \mathbf{e}$

$$
\begin{aligned}
\partial_{t} \mathbf{e}(t)= & -\boldsymbol{\Omega} \sin \left(\left(t-t^{\prime}\right) \boldsymbol{\Omega}\right) \mathbf{e}\left(t^{\prime}\right)+\cos \left(\left(t-t^{\prime}\right) \boldsymbol{\Omega}\right) \partial_{t} \mathbf{e}\left(t^{\prime}\right) \\
& +\left(t-t^{\prime}\right) \int_{0}^{1} \cos \left(\left(t-t^{\prime}\right)(1-\xi) \boldsymbol{\Omega}\right) \mathbf{G e}\left(t^{\prime}(1-\xi)+t \xi\right) \mathrm{d} \xi
\end{aligned}
$$

For an e-only formulation for the numerical scheme, we insert (3.1a) into (3.1b). Inserting the resulting expression for $\mathbf{e}_{n}^{+}$into (3.1c), a formula for $\mathbf{e}_{n+1}^{-}$is obtained. Finally, we substitute $\mathbf{e}_{n+1}^{-}$in (3.1d) and use (3.1a) once more to obtain

$$
\begin{aligned}
\mathbf{e}_{n+1}=- & \boldsymbol{\Omega} \sin (\tau \boldsymbol{\Omega}) \mathbf{p}_{n}+\cos (\tau \boldsymbol{\Omega}) \mathbf{e}_{n}+\tau \frac{1}{2}(\cos (\tau \boldsymbol{\Omega})+\mathrm{Id}) \psi_{E}\left(\frac{\tau}{2} \boldsymbol{\Omega}\right) \mathbf{C}_{B} \phi_{B}\left(\frac{\tau}{2} \boldsymbol{\Omega}\right) \mathbf{b}_{n} \\
& +\tau^{2} \frac{1}{4}(\cos (\tau \boldsymbol{\Omega})+\mathrm{Id}) \psi_{E}\left(\frac{\tau}{2} \boldsymbol{\Omega}\right) \mathbf{C}_{B} \phi_{B}\left(\frac{\tau}{2} \boldsymbol{\Omega}\right) \psi_{B}\left(\frac{\tau}{2} \boldsymbol{\Omega}\right) \mathbf{C}_{E} \phi_{E}\left(\frac{\tau}{2} \boldsymbol{\Omega}\right) \mathbf{e}_{n} .
\end{aligned}
$$

The filter functions $\psi_{i}, \phi_{i}, i \in\{E, B\}$, are even, and hence the matrix functions that are applied to $\mathbf{p}_{n}$ and $\mathbf{b}_{n}$ are uneven as functions of $\tau$, whereas the matrix functions that are applied to $\mathbf{e}_{n}$ are even in $\tau$. This observation results in the two-step formulation

$$
\begin{aligned}
\mathbf{e}_{n+1}-2 & \cos (\tau \boldsymbol{\Omega}) \mathbf{e}_{n}+\mathbf{e}_{n-1} \\
& =\tau^{2} \frac{1}{2}(\cos (\tau \boldsymbol{\Omega})+\mathrm{Id}) \psi_{E}\left(\frac{\tau}{2} \boldsymbol{\Omega}\right) \mathbf{C}_{B} \phi_{B}\left(\frac{\tau}{2} \boldsymbol{\Omega}\right) \psi_{B}\left(\frac{\tau}{2} \boldsymbol{\Omega}\right) \mathbf{C}_{E} \phi_{E}\left(\frac{\tau}{2} \boldsymbol{\Omega}\right) \mathbf{e}_{n} .
\end{aligned}
$$

To obtain a formulation close to the two-step form of [8, Chapter XIII], we use (5.1) and get rid of the filter functions "between" the two curl operators

$$
\mathbf{e}_{n+1}-2 \cos (\tau \boldsymbol{\Omega}) \mathbf{e}_{n}+\mathbf{e}_{n-1}=\tau^{2} \frac{1}{2}(\cos (\tau \boldsymbol{\Omega})+\mathrm{Id}) \psi_{E}\left(\frac{\tau}{2} \boldsymbol{\Omega}\right) \mathbf{G} \phi_{E}\left(\frac{\tau}{2} \boldsymbol{\Omega}\right) \mathbf{e}_{n} .
$$

Again with (5.1) the equations for $\mathbf{p}$ and $\mathbf{b}$ of the numerical scheme finally read

$$
\begin{gathered}
\mathbf{p}_{n+1}=\cos (\tau \boldsymbol{\Omega}) \mathbf{p}_{n}+\tau \operatorname{sinc}(\tau \boldsymbol{\Omega}) \mathbf{e}_{n}+\tau^{2} \frac{1}{2} \operatorname{sinc}(\tau \boldsymbol{\Omega}) \psi_{E}\left(\frac{\tau}{2} \boldsymbol{\Omega}\right) \mathbf{C}_{B} \mathbf{b}_{n} \\
-\tau^{3} \frac{1}{4} \operatorname{sinc}(\tau \boldsymbol{\Omega}) \psi_{E}\left(\frac{\tau}{2} \boldsymbol{\Omega}\right) \mathbf{G} \phi_{E}\left(\frac{\tau}{2} \boldsymbol{\Omega}\right) \mathbf{e}_{n},
\end{gathered}
$$

and

$$
\mathbf{b}_{n+1}=\mathbf{b}_{n}-\tau \frac{1}{2} \mathbf{C}_{E} \phi_{E}\left(\frac{\tau}{2} \boldsymbol{\Omega}\right)\left(\mathbf{e}_{n}+\mathbf{e}_{n+1}\right) .
$$

6.2. Error in the electric field. We want to apply [8, Theorem XIII.4.1] to estimate the error in the electric field. Unfortunately, this requires a distinct first time step, which our scheme (3.1) does not fulfill. To circumvent this problem we perturb the initial value for the derivative of the e-field, which then yields the correct scheme. For an estimate with the original initial values, we use a stability estimate for the exact solution.

The following theorem restates [8, Theorem XIII.4.1] adapted to the situation at hand.

THEOREM 6.1. Let $\boldsymbol{\Omega}$ and $\mathbf{G}$ be as in Assumption 4.1. Consider the solution of equation (6.1a) for the electric field by the method (6.7) with a step size $\tau \leq \tau_{0}$ for a sufficiently 
small $\tau_{0}$ independent of $\widetilde{\omega}$ with $\tau \widetilde{\omega} \geq c_{0}>0$, where $c_{0}$ is independent of $\tau$ and $\widetilde{\omega}$. We denote the exact solution by $\mathbf{e}^{\prime}(t)$ and the numerical solution by $\mathbf{e}_{n}{ }^{\prime}$. The first time step is computed via

$$
\mathbf{e}_{1}{ }^{\prime}=\cos (\tau \boldsymbol{\Omega}) \mathbf{e}_{0}{ }^{\prime}+\tau \operatorname{sinc}(\tau \boldsymbol{\Omega}) \dot{\mathbf{e}}_{0}{ }^{\prime}+\tau^{2} \frac{1}{4}(\cos (\tau \boldsymbol{\Omega})+\mathrm{Id}) \psi_{E}\left(\frac{\tau}{2} \boldsymbol{\Omega}\right) \mathbf{G} \phi_{E}\left(\frac{\tau}{2} \boldsymbol{\Omega}\right) \mathbf{e}_{0}{ }^{\prime},
$$

where

$$
\mathbf{e}_{0}{ }^{\prime}:=\mathbf{e}_{0} \quad \text { and } \quad \dot{\mathbf{e}}_{0}{ }^{\prime}:=\chi(\tau \boldsymbol{\Omega}) \mathbf{C}_{B} \mathbf{b}_{0}-\boldsymbol{\Omega}^{2} \mathbf{p}_{0}
$$

with

$$
\chi(z):=\frac{1}{2} \frac{\cos (z)+1}{\operatorname{sinc}(z)} \psi_{E}\left(\frac{1}{2} z\right)
$$

and $\mathbf{e}_{0}, \mathbf{b}_{0}$, and $\mathbf{p}_{0}$ are such that the conditions (4.3) hold true.

If the conditions (5.2a), (5.2b), (5.2c), and (5.2d) are satisfied with constants independent of $\widetilde{\omega}$ for the even entire filter functions $\psi_{E}, \phi_{E}: \mathbb{R}_{\geq 0} \rightarrow \mathbb{R}$ with $\psi_{E}(0)=\phi_{E}(0)=1$, then we obtain

$$
\left\|\mathbf{e}_{n}{ }^{\prime}-\mathbf{e}^{\prime}\left(t_{n}\right)\right\| \leq C \tau^{2} \quad \text { for } \quad t_{n}:=t_{0}+n \tau \leq T,
$$

with a constant $C$ independent of $n, \tau$, and $\tilde{\omega}$ but depending on $\left(T-t_{0}\right)$ and the constants $H_{0}, C_{g}, c_{0}$, and $C_{1}, \ldots, C_{3}$.

Proof. The filter functions of [8, Theorem XIII.4.1] are

$$
\psi(z):=\frac{1}{2}(\cos (z)+1) \psi_{E}\left(\frac{1}{2} z\right), \quad \phi(z):=\phi_{E}\left(\frac{1}{2} z\right), \quad \forall z \in \mathbb{C} .
$$

As $\mathbf{G}$ is symmetric we can write $\mathbf{G e}=\nabla U(\mathbf{e})$ with $U=\frac{1}{2} \mathbf{e}^{T} \mathbf{G e}$. From condition (5.2d) we obtain that $\chi$ is bounded by $C_{4}$. The factor $4 / C_{4}^{2}$ in the estimate for $\mathbf{C}_{B} \mathbf{b}_{0}$ in (4.3) guarantees the estimate for the initial oscillatory energy for the perturbed initial values. Hence, all assumptions of [8, Theorem XIII.4.1] are fulfilled, and its application completes the proof. (

REMARK 6.2. We carefully analyzed the proof of the second-order convergence result [8, Theorem XIII.4.1] using the strong filter assumptions. With the help of Ernst Hairer we could close a gap in the proof of [8, Theorem XIII.4.1]. It is the second-order convergence result that we have reformulated in Theorem 6.1. In the supplement of Theorem XIII.4.1 in [8], it is claimed that one would obtain

$$
\left\|\mathbf{e}_{n}{ }^{\prime}-\mathbf{e}^{\prime}\left(t_{n}\right)\right\| \leq C \tau,
$$

if only the weaker estimate $|\psi(z)| \leq C_{0}\left|\operatorname{sinc}\left(\frac{1}{2} z\right)\right|$ instead of $|\psi(z)| \leq C_{1} \operatorname{sinc}\left(\frac{1}{2} z\right)^{2}$ holds. In [8, Theorem XIII.4.1], a general nonlinearity $g$ is considered. Numerical experiments with a linear $g$, i.e., $g(\mathbf{e})=\mathbf{G e}$, as the one shown in Section 8.2 are a counterexample to this claim. It is the above weaker estimate that is fulfilled by the filter choice (5.3).

REMARK 6.3. By perturbing $\dot{\mathbf{e}}_{0}$ of (6.1b) to $\dot{\mathbf{e}}_{0}{ }^{\prime}$ of (6.11), we have

$$
\mathbf{e}_{n}=\mathbf{e}_{n}{ }^{\prime}, \quad \forall n \geq 0,
$$

when computing $\mathbf{e}_{n}$ with the original scheme (3.1) and $\mathbf{e}_{n}{ }^{\prime}$ with the method described in Theorem 6.1, thus we can replace the numerical solution there by the scheme (3.1). 
To control the perturbation we apply the stability estimate of Lemma 6.4 to the exact solution and obtain $\left\|\mathbf{e}(t)-\mathbf{e}^{\prime}(t)\right\| \leq C \tau^{2}$ again with $C$ independent of $n, \tau$, and $\widetilde{\omega}$.

Lemma 6.4. Consider the exact solution $\Delta \mathbf{e}(t)$ of (6.1a) with initial values $\Delta \mathbf{e}\left(t_{0}\right)=0$ and $\partial_{t} \Delta \mathbf{e}\left(t_{0}\right)=(\operatorname{Id}-\chi(\tau \boldsymbol{\Omega})) \mathbf{C}_{B} \mathbf{b}_{0}$ for a given $\mathbf{b}_{0}$ and $\chi$ from (6.12) such that Assumption 4.1 holds.

If the filter functions $\psi_{E}, \phi_{E}: \mathbb{R}_{\geq 0} \rightarrow \mathbb{R}$ are entire functions with $\psi_{E}(0)=\phi_{E}(0)=1$ satisfying (5.1) and (5.2e) with a constant $C_{5}$ independent of $\widetilde{\omega}$, then

$$
\|\Delta \mathbf{e}(t)\| \leq C \tau^{2}
$$

with a constant $C$ independent of $n, \tau$, and $\omega$ but depending on $\left(T-t_{0}\right), C_{5}$, and $H_{0}$.

Proof. By Assumption 4.1 the matrices $(-\mathbf{G})$ and $\boldsymbol{\Omega}^{2}$ are both symmetric positive semidefinite and so are their sum, and we can define the symmetric positive semi-definite matrix $\mathbf{B}:=\sqrt{\Omega^{2}-\mathbf{G}}$. Using the matrix sinc function, the exact solution is

$$
\begin{aligned}
\Delta \mathbf{e}(t) & =\cos \left(\left(t-t_{0}\right) \mathbf{B}\right) \Delta \mathbf{e}\left(t_{0}\right)+\left(t-t_{0}\right) \operatorname{sinc}\left(\left(t-t_{0}\right) \mathbf{B}\right) \partial_{t} \Delta \mathbf{e}\left(t_{0}\right) \\
& =\left(t-t_{0}\right) \operatorname{sinc}\left(\left(t-t_{0}\right) \mathbf{B}\right)(\operatorname{Id}-\chi(\tau \boldsymbol{\Omega})) \mathbf{C}_{B} \mathbf{b}_{0} .
\end{aligned}
$$

Since $|z \operatorname{sinc}(z)| \leq 1, z \geq 0$, we only have to control the real part. Condition (5.2e) yields

$$
|1-\chi(z)| \leq C_{5} z^{2} \quad \Rightarrow \quad\left\|(\operatorname{Id}-\chi(\tau \boldsymbol{\Omega})) \mathbf{C}_{B} \mathbf{b}_{0}\right\| \leq C_{5} \tau^{2}\left\|\boldsymbol{\Omega}^{2} \mathbf{C}_{B} \mathbf{b}_{0}\right\| .
$$

This gives the desired bound for $C=\left(T-t_{0}\right) C_{5} \sqrt{H_{0}}$.

By combining Theorem 6.1 and Lemma 6.4, the error estimate for the electric field is obtained:

THEOREM 6.5. Let $\mathbf{G}, \boldsymbol{\Omega}$ and the initial values $\mathbf{p}_{0}, \mathbf{e}_{0}$, and $\mathbf{b}_{0}$ be as in Assumptions 4.1 and 4.2. Consider the numerical solution of (2.2) with the scheme (3.1) with step size $\tau$ satisfying $\tau \leq \tau_{0}$ for sufficiently small $\tau_{0}$ independent of $\widetilde{\omega}$ and $\tau \widetilde{\omega} \geq c_{0}>0$ with $c_{0}$ independent of $\tau$ and $\widetilde{\omega}$. Denote the exact solution for the electric field by $\mathbf{e}(t)$ and the numerical one by $\mathbf{e}_{n}$.

If the filter functions satisfy (5.1) and the assumptions (5.2a)-(5.2e) with constants independent of $\widetilde{\omega}$, then

$$
\left\|\mathbf{e}_{n}-\mathbf{e}\left(t_{n}\right)\right\| \leq C \tau^{2} \quad \text { for } \quad t_{n}:=t_{0}+n \tau \leq T
$$

with a constant $C$ independent of $n, \tau$, and $\omega$ but depending on $\left(T-t_{0}\right)$ and the constants $H_{0}, C_{g}, c_{0}$, and $C_{1}, \ldots, C_{5}$.

Proof. By Remark 6.3 the scheme (3.1) with the adjusted initial value (6.11) with (6.12) is the same as the two-step scheme with the first step (6.10) from Theorem 6.1. We again denote the perturbed exact solution by $\mathbf{e}^{\prime}(t)$. Since $\mathbf{e}\left(t_{0}\right)-\mathbf{e}^{\prime}\left(t_{0}\right)=0=\Delta \mathbf{e}\left(t_{0}\right)$ and $\partial_{t} \mathbf{e}\left(t_{0}\right)-\partial_{t} \mathbf{e}^{\prime}\left(t_{0}\right)=\partial_{t} \Delta \mathbf{e}\left(t_{0}\right)$ from Lemma 6.4, we have $\left\|\Delta \mathbf{e}\left(t_{n}\right)\right\| \leq C \tau^{2}$. From this and with Theorem 6.1 we then obtain

$$
\left\|\mathbf{e}_{n}-\mathbf{e}\left(t_{n}\right)\right\| \leq\left\|\mathbf{e}_{n}^{\prime}-\mathbf{e}^{\prime}\left(t_{n}\right)\right\|+\left\|\Delta \mathbf{e}\left(t_{n}\right)\right\| \leq C \tau^{2} .
$$

The constant $C$ has the stated dependencies.

6.3. Error in the magnetic flux. To get an estimate for the error in the magnetic flux $\mathbf{b}$, the filter assumption (5.2f) is needed.

THEOREM 6.6. Suppose the assumptions of Theorem 6.5 hold and additionally assume that (5.2f) holds with $C_{6}$ independent of $\widetilde{\omega}$. Then for $t_{n}:=t_{0}+n \tau \leq T$ we obtain

$$
\left\|\mathbf{b}_{n}-\mathbf{b}\left(t_{n}\right)\right\| \leq C \tau^{2}
$$


with a constant $C$ independent of $n, \tau$, and $\omega$ but depending on $\left(T-t_{0}\right)$ and the constants $H_{0}, C_{g}, c_{0}$, and $C_{1}, \ldots, C_{6}$.

Proof. From equations (2.2c) and (6.9), we obtain the recursion for the error in $\mathbf{b}$

$$
\mathbf{b}\left(t_{n+1}\right)-\mathbf{b}_{n+1}=\mathbf{b}\left(t_{n}\right)-\mathbf{b}_{n}-\frac{\tau}{2} \mathbf{C}_{E}\left(2 \int_{0}^{1} \mathbf{e}\left(t_{n}+\tau s\right) \mathrm{d} s-\phi_{E}\left(\frac{\tau}{2} \boldsymbol{\Omega}\right)\left(\mathbf{e}_{n}+\mathbf{e}_{n+1}\right)\right) .
$$

Applying the variation of constants formula (6.5) to (6.1a) for the argument $t_{n}+\tau s=t_{n+1}-(1-\tau) s$ to expand around $t_{n}$ and at the same time around $t_{n+1}$, we obtain for the term in parentheses

$$
\begin{aligned}
& 2 \int_{0}^{1} \mathbf{e}\left(t_{n}+\tau s\right) \mathrm{d} s-\phi_{E}\left(\frac{\tau}{2} \boldsymbol{\Omega}\right)\left(\mathbf{e}_{n}+\mathbf{e}_{n+1}\right) \\
& =\left(\int_{0}^{1} \cos (\tau s \boldsymbol{\Omega}) \mathrm{d} s-\phi_{E}\left(\frac{\tau}{2} \boldsymbol{\Omega}\right)\right) \mathbf{e}\left(t_{n}\right)+\left(\int_{0}^{1} \cos (\tau(s-1) \boldsymbol{\Omega}) \mathrm{d} s-\phi_{E}\left(\frac{\tau}{2} \boldsymbol{\Omega}\right)\right) \mathbf{e}\left(t_{n+1}\right) \\
& \quad+\tau\left(\int_{0}^{1} s \operatorname{sinc}(\tau s \boldsymbol{\Omega}) \mathrm{d} s \partial_{t} \mathbf{e}\left(t_{n}\right)+\int_{0}^{1}(s-1) \operatorname{sinc}(\tau(s-1) \boldsymbol{\Omega}) \mathrm{d} s \partial_{t} \mathbf{e}\left(t_{n+1}\right)\right) \\
& \quad+\tau^{2}\left(\int_{0}^{1} s^{2} I_{n}^{+}(\tau, s) \mathrm{d} s+\int_{0}^{1}(1-s)^{2} I_{n+1}^{-}(\tau, s) \mathrm{d} s\right) \\
& \quad+\phi_{E}\left(\frac{\tau}{2} \boldsymbol{\Omega}\right)\left(\left(\mathbf{e}\left(t_{n}\right)-\mathbf{e}_{n}\right)+\left(\mathbf{e}\left(t_{n+1}\right)-\mathbf{e}_{n+1}\right)\right),
\end{aligned}
$$

where $I_{n}^{+}$and $I_{n+1}^{-}$are bounded independently of $\widetilde{\omega}$ containing the convolution terms of the variation of constants formula. Here we use the boundedness of sinc and the bounds for $\mathbf{e}(t)$ from (6.3).

Computing the integrals and adding up the errors of all time steps yields

$$
\begin{aligned}
& \mathbf{b}\left(t_{n}\right)-\mathbf{b}_{n}= \\
& -\frac{\tau}{2} \mathbf{C}_{E} \sum_{l=0}^{n-1}\left[\left(\operatorname{sinc}(\tau \boldsymbol{\Omega})-\phi_{E}\left(\frac{\tau}{2} \boldsymbol{\Omega}\right)\right) \mathbf{e}\left(t_{l}\right)+\left(\operatorname{sinc}(\tau \boldsymbol{\Omega})-\phi_{E}\left(\frac{\tau}{2} \boldsymbol{\Omega}\right)\right) \mathbf{e}\left(t_{l+1}\right)\right] \\
& -\frac{\tau^{2}}{2} \mathbf{C}_{E} \sum_{l=0}^{n-1}\left[\operatorname{cosc}(\tau \boldsymbol{\Omega}) \partial_{t} \mathbf{e}\left(t_{l}\right)-\operatorname{cosc}(\tau \boldsymbol{\Omega}) \partial_{t} \mathbf{e}\left(t_{l+1}\right)\right] \\
& -\frac{\tau^{3}}{2} \mathbf{C}_{E} \sum_{l=0}^{n-1}\left[\int_{0}^{1} s^{2} I_{l}^{+}(\tau, s) \mathrm{d} s+\int_{0}^{1}(1-s)^{2} I_{l+1}^{-}(\tau, s) \mathrm{d} s\right] \\
& -\frac{\tau}{2} \mathbf{C}_{E} \sum_{l=0}^{n-1} \phi_{E}\left(\frac{\tau}{2} \boldsymbol{\Omega}\right)\left[\left(\mathbf{e}\left(t_{l}\right)-\mathbf{e}_{l}\right)+\left(\mathbf{e}\left(t_{l+1}\right)-\mathbf{e}_{l+1}\right)\right]
\end{aligned}
$$

with the even entire function $\operatorname{cosc}:=z \mapsto \int_{0}^{1} \cos ((1-\xi) z) \xi \mathrm{d} \xi$ satisfying the identity $z^{2} \operatorname{cosc}(z)=1-\cos (z)$ and the bound $|\operatorname{cosc}(z)| \leq \frac{1}{2}, z \in \mathbb{R}$.

We use the bound (5.2b) for $\phi_{E}$, the bound for $\mathbf{C}_{E}$, and the $\mathcal{O}\left(\tau^{2}\right)$-estimate of Theorem 6.5 to bound (6.14d) by $C \tau^{2}$, where we lose one factor $\tau$ due to summing up. The bound for (6.14c) follows from the boundedness of $I_{l}^{ \pm}$. (6.14b) is a telescopic sum, so we do not lose a $\tau$ by summation. The boundedness of the Hamiltonian in (6.2) yields a bound for $\partial_{t} \mathbf{e}(t)$. The boundedness of cosc then yields the second-order estimate for (6.14b). 
To control (6.14a) we apply the variation of constants formula (6.5) with $t^{\prime}=t_{0}$ to obtain $\mathbf{e}\left(t_{l}\right)$ :

$$
\begin{gathered}
\text { (6.14a) }=\frac{\tau}{2} \mathbf{C}_{E} \sum_{l=0}^{n-1}\left[\operatorname{sinc}(\tau \boldsymbol{\Omega})-\phi_{E}\left(\frac{\tau}{2} \boldsymbol{\Omega}\right)\right]\left(\mathbf{e}\left(t_{l}\right)+\mathbf{e}\left(t_{l+1}\right)\right) \\
=\frac{\tau}{2} \mathbf{C}_{E} \sum_{l=0}^{n-1}\left[\operatorname{sinc}(\tau \boldsymbol{\Omega})-\phi_{E}\left(\frac{\tau}{2} \boldsymbol{\Omega}\right)\right][\cos (l \tau \boldsymbol{\Omega})+\cos ((l+1) \tau \boldsymbol{\Omega})] \mathbf{e}_{0} \\
+\frac{\tau}{2} \mathbf{C}_{E} \sum_{l=0}^{n-1}\left[\operatorname{sinc}(\tau \boldsymbol{\Omega})-\phi_{E}\left(\frac{\tau}{2} \boldsymbol{\Omega}\right)\right] \times \\
+\tau \mathbf{C}_{E} \sum_{l=0}^{n}\left[\operatorname{sinc}(\tau \boldsymbol{\Omega})-\phi_{E}\left(\frac{\tau}{2} \boldsymbol{\Omega}\right)\right] \times \\
\quad\left[l^{2} \tau^{2} \int_{0}^{1}(1-\xi) \operatorname{sinc}(l \tau(1-\xi) \boldsymbol{\Omega}) \mathbf{G e}\left(t_{0}+\tau l \xi\right)\right],
\end{gathered}
$$

where the prime in the summation indicates that the first and last terms are weighted by $\frac{1}{2}$. At first sight the norm of each of the three terms $(6.15 \mathrm{a}, \mathrm{b}, \mathrm{c})$ seems to be of order $\mathcal{O}(1)$.

To show that they are actually of order $\mathcal{O}\left(\tau^{2}\right)$, we use the identities

$\cos (l z)=\frac{\sin \left(\left(l+\frac{1}{2}\right) z\right)-\sin \left(\left(l-\frac{1}{2}\right) z\right)}{2 \sin \left(\frac{1}{2} z\right)}, \quad l \operatorname{sinc}(l z)=-\frac{\cos \left(\left(l+\frac{1}{2}\right) z\right)-\cos \left(\left(l-\frac{1}{2}\right) z\right)}{2 z \sin \left(\frac{1}{2} z\right)}$.

These allow us to simplify the sum of cosines and sincs in (6.15a) and (6.15b), respectively, by using

$$
\begin{aligned}
\left(\operatorname{sinc}(z)-\phi_{E}\left(\frac{1}{2} z\right)\right) & \left(\sum_{l=0}^{n-1} \cos (l z)+\sum_{l=1}^{n} \cos (l z)\right) \\
& =\sin (n z) \cos \left(\frac{1}{2} z\right) z \frac{\operatorname{sinc}(z)-\phi_{E}\left(\frac{1}{2} z\right)}{z \sin \left(\frac{1}{2} z\right)}, \\
\left(\operatorname{sinc}(z)-\phi_{E}\left(\frac{1}{2} z\right)\right) & \left(\sum_{l=0}^{n-1} l \operatorname{sinc}(l z)+\sum_{l=1}^{n} l \operatorname{sinc}(l z)\right) \\
& =-(\cos (n z)-1) \cos \left(\frac{1}{2} z\right) \frac{\operatorname{sinc}(z)-\phi_{E}\left(\frac{1}{2} z\right)}{z \sin \left(\frac{1}{2} z\right)} .
\end{aligned}
$$

The factor of the trigonometric functions in front of the fractions on the right-hand sides above are bounded such that it suffices to control the expression

$$
\chi_{0}(z):=\frac{\operatorname{sinc}(z)-\phi_{E}\left(\frac{1}{2} z\right)}{z \sin \left(\frac{1}{2} z\right)} .
$$

This is the place where we finally use the new filter assumption (5.2f) to obtain

$$
\left|\chi_{0}(z)\right| \leq C_{6}
$$

such that potential new singularities are controlled. We obtain

$$
\|(6.15 \mathrm{a})\| \leq \frac{\tau}{2} C_{c}\left\|\sin (n \tau \boldsymbol{\Omega}) \cos \left(\frac{1}{2} \tau \boldsymbol{\Omega}\right)\right\|\left\|\chi_{0}(\tau \boldsymbol{\Omega}) \tau \boldsymbol{\Omega} \mathbf{e}_{0}\right\| \leq \frac{\tau}{2} C_{c} C_{6} \tau \sqrt{\frac{2}{3} H_{0}}
$$


since $\sin$ and $\cos$ are bounded by one, $\chi_{0}$ by $C_{6}$, and $\left\|\boldsymbol{\Omega} \mathbf{e}_{0}\right\|$ by $\frac{2}{3} H_{0}$; cf. (4.3). Likewise we have

$$
\|(6.15 \mathrm{~b})\| \leq \frac{1}{2} \tau^{2} C_{c}\left\|(\cos (n \tau \boldsymbol{\Omega})-\mathrm{Id}) \cos \left(\frac{1}{2} \tau \boldsymbol{\Omega}\right)\right\|\left\|\chi_{0}(\tau \boldsymbol{\Omega}) \dot{\mathbf{e}}_{0}\right\| \leq \frac{1}{2} \tau^{2} C_{c} 2 C_{6} \sqrt{2 H_{0}},
$$

where $\dot{\mathbf{e}}_{0}$ is bounded by the Hamiltonian in (6.2).

This way we used the filter function $\phi_{E}$ to filter periodic singularities. This is the reason why we need sinc terms on the right-hand side of the filter assumptions. For the remainder we use it to filter out higher-order singularities in a neighborhood of zero that leads to factors of $z$ on the right-hand side in the filter assumptions.

It remains to bound the integral term of the summand (6.15c), that is, we need an $\mathcal{O}(1)$ bound for

$$
J_{l}:=\tau \mapsto l^{2} \vartheta_{0}(\tau \boldsymbol{\Omega}) \int_{0}^{1}(1-\xi) \operatorname{sinc}(l \tau(1-\xi) \boldsymbol{\Omega}) f(\xi) \mathrm{d} \xi
$$

with

$$
f:=\xi \mapsto \mathbf{G e}\left(t_{0}+\tau l \xi\right)
$$

and the auxiliary functions

$$
\vartheta_{i}(z):=\frac{\operatorname{sinc}(z)-\phi_{E}\left(\frac{1}{2} z\right)}{z^{i}}, \quad i \in\{0,1,2\} .
$$

These functions $\vartheta_{i}$ satisfy the relations

$$
z \vartheta_{i}(z)=\vartheta_{i-1}(z), \quad i \in\{1,2\}
$$

where the first one in turn yields

$$
l \vartheta_{0}(z)(1-\xi) \operatorname{sinc}(l(1-\xi) z)=\vartheta_{1}(z) \sin (l(1-\xi) z) .
$$

Applying the filter assumption (5.2f) directly gives an $\mathcal{O}(1)$-bound for $\vartheta_{1}$, which in turn leads to a $\mathcal{O}(n)$-bound for $J_{l}$, for $l=1, \ldots, n$, and thus to a first-order estimate for the magnetic flux.

To improve this estimate we make use of the identity $z \operatorname{sinc}(z)=\sin (z)$, which gives an even sharper estimate for the filtering abilities of $\phi_{E}$ by

$$
\left|\operatorname{sinc}(z)-\phi_{E}\left(\frac{1}{2} z\right)\right| \leq C_{6}\left|\frac{1}{2} z^{2} \operatorname{sinc}\left(\frac{1}{2} z\right)\right| \leq \frac{1}{2} C_{6} z^{2}
$$

and thus an $\mathcal{O}(1)$-bound for $\vartheta_{2}$ since the sinc-function is bounded by one.

To make use of this estimate we employ

$$
\vartheta_{1}(z) l \sin (l(1-\xi) z) \stackrel{(6.17)}{=} \vartheta_{2}(z) l z \sin (l(1-\xi) z)=\frac{\partial}{\partial \xi} \vartheta_{2}(z) \cos (l(1-\xi) z) .
$$

Integration by parts of $J_{l}$ yields

$$
\begin{aligned}
\int_{0}^{1} \vartheta_{1}(z) l \sin (l(1-\xi) z) f\left(t_{0}+l \tau \xi\right) \mathrm{d} \xi= & {\left[\left.\vartheta_{2}(z) \cos (l(1-\xi) z) f\left(t_{0}+l \tau \xi\right)\right|_{\xi=0} ^{\xi=1}\right.} \\
& -\int_{0}^{1} \vartheta_{2}(z) \cos (l(1-\xi) z) \frac{\partial f}{\partial \xi}\left(t_{0}+l \tau \xi\right) l \tau \mathrm{d} \xi
\end{aligned}
$$


Since by definition of $f$ in (6.16), the derivative $\frac{\mathrm{d}}{\mathrm{d} \xi} f$ is given by

$$
\frac{\mathrm{d}}{\mathrm{d} \xi} f: \xi \mapsto l \tau \mathbf{G} \partial_{t} \mathbf{e}\left(t_{0}+\tau l \xi\right)
$$

and this applies to $J_{l}$ by

$$
\begin{aligned}
J_{l}(\tau)=\vartheta_{2}(\tau \boldsymbol{\Omega}) & \mathbf{G e}\left(t_{0}+l \tau\right)-\vartheta_{2}(\tau \boldsymbol{\Omega}) \cos (l \tau \boldsymbol{\Omega}) \mathbf{G e}_{0} \\
& -\int_{0}^{1} \vartheta_{2}(\tau \boldsymbol{\Omega}) \cos (l \tau(1-\xi) \boldsymbol{\Omega}) l \tau \mathbf{G} \partial_{t} \mathbf{e}\left(t_{0}+l \tau \xi\right) \mathrm{d} \xi .
\end{aligned}
$$

The boundedness of $\mathbf{G}$ in Assumption 4.1 and the stability estimates in (6.2) and (6.3) allow us to control Ge and $\mathbf{G} \partial_{t} \mathbf{e}$. All the matrix functions are bounded, and $l \tau \leq T-t_{0}$ such that $J_{l}(\tau)$ is of the order $\mathcal{O}(1)$ with a constant independent of $\widetilde{\omega}$. This concludes the proof for the error in the magnetic flux.

REMARK 6.7. By choosing $\phi_{E}(z)=\operatorname{sinc}(2 z)$, the left-hand side of (5.2f) and the term (6.14a) vanish, thus one may set $C_{6}=0$ and the above proof is simplified drastically. It is shown in [12] that the choice $\phi_{E}(z)=\operatorname{sinc}(2 z)$ is indeed a valid choice and respects the filter conditions (5.2b), (5.2c), (5.2f) and (5.2h).

6.4. Error in the impulse. To conclude the proof of the main result, Theorem 5.1, we have to verify the corresponding estimate for the error in the impulse. This is where the last two assumptions on the filter functions $(5.2 \mathrm{~g})$ and $(5.2 \mathrm{~h})$ enter.

THEOREM 6.8. Suppose that the assumptions of Theorem 6.6 hold. If $(5.2 \mathrm{~g})$ and $(5.2 \mathrm{~h})$ hold with $C_{7}$ and $C_{8}$ independent of $\widetilde{\omega}$, then

$$
\left\|\mathbf{p}_{n}-\mathbf{p}\left(t_{n}\right)\right\| \leq C \tau^{2} \quad \text { for } \quad t_{n}:=t_{0}+n \tau \leq T,
$$

with a constant $C$ independent of $n, \tau$, and $\omega$ but depending on $\left(T-t_{0}\right)$ and the constants $H_{0}, C_{g}, c_{0}$, and $C_{1}, \ldots, C_{8}$.

Proof. We start by expressing the impulse with the fundamental theorem of calculus applied to the differential equation for the impulse (2.2a). Applying (6.5) gives a formula for the exact solution of the electric field. The numerical solution is expressed by (6.8). Then the error in the $(n+1)$ st step reads

$$
\begin{aligned}
\mathbf{p}\left(t_{n+1}\right)-\mathbf{p}_{n+1}=\mathbf{p}\left(t_{n}\right)+ & \tau\left(\int_{0}^{1} \cos (\tau s \boldsymbol{\Omega}) \mathrm{d} s \mathbf{e}\left(t_{n}\right)-\operatorname{sinc}(\tau \boldsymbol{\Omega}) \mathbf{e}_{n}\right) \\
& +\tau \int_{0}^{1} \tau s \operatorname{sinc}(\tau s \boldsymbol{\Omega}) \mathrm{d} s \partial_{t} \mathbf{e}\left(t_{n}\right)-\cos (\tau \boldsymbol{\Omega}) \mathbf{p}_{n} \\
& -\frac{\tau^{2}}{2} \operatorname{sinc}(\tau \boldsymbol{\Omega}) \psi_{E}\left(\frac{\tau}{2} \boldsymbol{\Omega}\right) \mathbf{C}_{B} \mathbf{b}_{n}+\tau^{3} I_{n}(\tau) \\
=\mathbf{p}\left(t_{n}\right) & +\tau \operatorname{sinc}(\tau \boldsymbol{\Omega})\left(\mathbf{e}\left(t_{n}\right)-\mathbf{e}_{n}\right)+\tau^{3} I_{n}(\tau) \\
& +\tau^{2}\left(\operatorname{cosc}(\tau \boldsymbol{\Omega}) \mathbf{C}_{B} \mathbf{b}\left(t_{n}\right)-\frac{1}{2} \operatorname{sinc}(\tau \boldsymbol{\Omega}) \psi_{E}\left(\frac{\tau}{2} \boldsymbol{\Omega}\right) \mathbf{C}_{B} \mathbf{b}_{n}\right) \\
& -\tau^{2} \boldsymbol{\Omega}^{2} \operatorname{cosc}(\tau \boldsymbol{\Omega}) \mathbf{p}\left(t_{n}\right)-\cos (\tau \boldsymbol{\Omega}) \mathbf{p}_{n},
\end{aligned}
$$

where

$$
\begin{aligned}
I_{n}(\tau):=\int_{0}^{1} s^{2} \int_{0}^{1}(1-\xi) \operatorname{sinc}( & \tau s(1-\xi) \boldsymbol{\Omega}) \mathbf{G e}\left(t_{n}+\tau s \xi\right) \mathrm{d} \xi \mathrm{d} s \\
& -\frac{1}{4} \operatorname{sinc}(\tau \boldsymbol{\Omega}) \psi_{E}\left(\frac{\tau}{2} \boldsymbol{\Omega}\right) \mathbf{G} \phi_{E}\left(\frac{\tau}{2} \boldsymbol{\Omega}\right) \mathbf{e}_{n}
\end{aligned}
$$


The cosc-function was already used in (6.14b) for the estimate for $\mathbf{b}$ and can also be written as an integral over $\xi \operatorname{sinc}(\xi z)$. The filter estimate (5.2g) yields the boundedness of $\psi_{E}$. With the estimate for $\mathbf{e}$ in Theorem 6.5, $\left\|\mathbf{e}_{n}\right\| \leq\left\|\mathbf{e}\left(t_{n}\right)\right\|+\left\|\mathbf{e}_{n}-\mathbf{e}\left(t_{n}\right)\right\|$, and the stability estimate for the electric field (6.3), we obtain

$$
\left\|I_{n}(\tau)\right\| \leq C_{I}
$$

with a constant $C_{I}$ independent of $\widetilde{\omega}$ since $\tau \leq \tau_{0}$. For (6.18b) we use $z^{2} \operatorname{cosc}(z)=1-\cos (z)$ to retrieve

$$
-\tau^{2} \boldsymbol{\Omega}^{2} \operatorname{cosc}(\tau \boldsymbol{\Omega}) \mathbf{p}\left(t_{n}\right)-\cos (\tau \boldsymbol{\Omega}) \mathbf{p}_{n}=\cos (\tau \boldsymbol{\Omega})\left(\mathbf{p}\left(t_{n}\right)-\mathbf{p}_{n}\right)-\mathbf{p}\left(t_{n}\right) .
$$

For (6.18a), we get analogously with $\operatorname{cosc}(2 z)=\frac{1}{2} \operatorname{sinc}^{2}(z)$

$$
\begin{array}{r}
\tau^{2}\left(\operatorname{cosc}(\tau \boldsymbol{\Omega}) \mathbf{C}_{B} \mathbf{b}\left(t_{n}\right)-\frac{1}{2} \operatorname{sinc}(\tau \boldsymbol{\Omega}) \psi_{E}\left(\frac{\tau}{2} \boldsymbol{\Omega}\right) \mathbf{C}_{B} \mathbf{b}_{n}\right) \\
=\frac{\tau^{2}}{2}\left(\left(\operatorname{sinc}^{2}\left(\frac{\tau}{2} \boldsymbol{\Omega}\right)-\operatorname{sinc}(\tau \boldsymbol{\Omega}) \psi_{E}\left(\frac{\tau}{2} \boldsymbol{\Omega}\right)\right) \mathbf{C}_{B} \mathbf{b}\left(t_{n}\right)\right. \\
\left.\quad+\operatorname{sinc}(\tau \boldsymbol{\Omega}) \psi_{E}\left(\frac{\tau}{2} \boldsymbol{\Omega}\right) \mathbf{C}_{B}\left(\mathbf{b}\left(t_{n}\right)-\mathbf{b}_{n}\right)\right) .
\end{array}
$$

We next define the auxiliary function

$$
J_{n}(\tau):=\tau\left(\operatorname{sinc}(\tau \boldsymbol{\Omega})\left(\mathbf{e}\left(t_{n}\right)-\mathbf{e}_{n}\right)+\tau \operatorname{sinc}(\tau \boldsymbol{\Omega}) \psi_{E}\left(\frac{\tau}{2} \boldsymbol{\Omega}\right) \mathbf{C}_{B}\left(\mathbf{b}\left(t_{n}\right)-\mathbf{b}_{n}\right)+\tau^{2} I_{n}(\tau)\right) .
$$

This, the boundedness of sinc, $\psi_{E}$, and $\mathbf{C}_{B}$, and the error estimates for $\mathbf{e}$ and $\mathbf{b}$ from Theorems 6.5 and 6.6 yield the second-order estimate

$$
\left\|J_{n}(\tau)\right\| \leq C_{E} \tau^{2}+\tau C_{7} C_{c} C_{B} \tau^{2}+C_{I} \tau^{2}=: C_{J} \tau^{2}
$$

for $J_{n}(\tau)$. Resolving the recursion in (6.18) we obtain the summed error

$$
\begin{aligned}
\mathbf{p}\left(t_{n}\right)-\mathbf{p}_{n}= & \tau \sum_{l=0}^{n} \cos ^{l}(\tau \boldsymbol{\Omega}) J_{n-l-1}(\tau) \\
& +\frac{\tau^{2}}{2} \sum_{l=0}^{n} \cos ^{l}(\tau \boldsymbol{\Omega})\left(\operatorname{sinc}^{2}\left(\frac{\tau}{2} \boldsymbol{\Omega}\right)-\operatorname{sinc}(\tau \boldsymbol{\Omega}) \psi_{E}\left(\frac{\tau}{2} \boldsymbol{\Omega}\right)\right) \mathbf{C}_{B} \mathbf{b}\left(t_{n-l-1}\right) .
\end{aligned}
$$

The first summand with $J_{n}(\tau)$ and the leading factor of $\tau$ is of the proper order due to (6.19). The second summand seems to be of a too low order to succeed with a global error proof of a second-order convergence. We have to use the trigonometric identity

$$
\cos ^{n}(z)=\frac{\cos ^{n+1}(z)-\cos ^{n}(z)}{-2 \sin ^{2}\left(\frac{1}{2} z\right)}
$$

and the filtering abilities of $\psi_{E}$ to avoid a summation of errors. With the help of partial summation

$$
\sum_{l=0}^{n-1}\left(f_{l+1}-f_{l}\right) g_{l}=\sum_{l=0}^{n-1} f_{l}\left(g_{l-1}-g_{l}\right)+f_{n} g_{n-1}-f_{0} g_{-1}
$$


with $f_{l}:=\frac{\cos ^{l}(z)}{-2 \sin ^{2}\left(\frac{1}{2} z\right)}$ and $g_{l}:=\mathbf{C}_{B} \mathbf{b}\left(t_{n-l-1}\right)$, the trigonometric identity yields

$$
\begin{aligned}
\left(\operatorname{sinc}^{2}\left(\frac{1}{2} z\right)-\operatorname{sinc}(z) \psi_{E}\left(\frac{1}{2} z\right)\right) \sum_{l=0}^{n-1} \cos ^{l}(z) \mathbf{C}_{B} \mathbf{b}\left(t_{n-l-1}\right) \\
=\frac{\operatorname{sinc}^{2}\left(\frac{1}{2} z\right)-\operatorname{sinc}(z) \psi_{E}\left(\frac{1}{2} z\right)}{-2 \sin ^{2}\left(\frac{1}{2} z\right)} \times \\
\quad\left(\sum_{l=1}^{n} \cos ^{l}(z) \mathbf{C}_{B}\left(\mathbf{b}\left(t_{n-l}\right)-\mathbf{b}\left(t_{n-l-1}\right)\right)+\cos ^{n}(z) \mathbf{C}_{B} \mathbf{b}_{0}-\mathbf{C}_{B} \mathbf{b}\left(t_{n}\right)\right) .
\end{aligned}
$$

The filter assumption $(5.2 \mathrm{~h})$ gives us the estimate

$$
\left|\frac{\operatorname{sinc}^{2}\left(\frac{1}{2} z\right)-\operatorname{sinc}(z) \psi_{E}\left(\frac{1}{2} z\right)}{-2 \sin ^{2}\left(\frac{1}{2} z\right)}\right| \leq \frac{1}{2} C_{8}
$$

for the singularities that appear in (6.21), and thus

$$
\| \text { r.h.s. of }(6.21) \| \leq \frac{1}{2} C_{8}\left(\sum_{l=0}^{n-1}\left\|\mathbf{C}_{B}\left(\mathbf{b}\left(t_{n-l}\right)-\mathbf{b}\left(t_{n-l-1}\right)\right)\right\|+\widetilde{C}\right)
$$

using the boundedness of the magnetic flux (6.4) to estimate the boundary terms $\mathbf{C}_{B} \mathbf{b}_{0}$ and $\mathbf{C}_{B} \mathbf{b}\left(t_{n}\right)$ with a constant $\widetilde{C}$ independent of $\widetilde{\omega}$. Since the boundary terms appear only once, it is sufficient that they are of order $\mathcal{O}(1)$.

To generate the last factor of $\tau$ we once more need to apply the fundamental theorem of calculus, this time to the exact solution of the magnetic flux and substitute the right-hand side of the differential equation for $\mathbf{b}(2.2 \mathrm{c})$ into the time derivative:

$$
\begin{aligned}
& \left\|\mathbf{C}_{B}\left(\mathbf{b}\left(t_{n-l}\right)-\mathbf{b}\left(t_{n-l-1}\right)\right)\right\| \\
& \quad=\left\|\mathbf{C}_{B}\left(\mathbf{b}\left(t_{n-l-1}\right)-\tau \int_{0}^{1} \mathbf{C}_{E} \mathbf{e}\left(t_{n-l-1}+\tau \xi\right) \mathrm{d} \xi-\mathbf{b}\left(t_{n-l-1}\right)\right)\right\| \leq \tau \widehat{C},
\end{aligned}
$$

with another constant $\widehat{C}$ independent of $\widetilde{\omega}$ using the boundedness of $\mathbf{e}(t)$. The $\tau^{2}$-factor in front of the second sum in the error formula (6.20) is thus sufficient for the global second-order estimate.

7. Multiple high frequencies. Consider now the case of multiple frequencies, i.e., let us assume that $\Omega$ is a positive semi-definite matrix and that $\omega$ is a bound for its largest eigenvalue. Modifying the results and the proof of [6], a proof for the second-order error estimate for the triple splitting method was obtained by [2], thus generalizing the results here using a different technique.

To extend our arguments to this case, the only ingredient that is required in the convergence proof is a replacement for Theorem 6.1. We can use [6, Theorem 1] without modifications by writing their scheme as a two-step formulation for the solution, getting rid of its derivative. Again we have to perturb the initial values to adjust to the situation at hand.

We use the multistep form (6.7) with the distinct first step (6.10) for the perturbed initial values (6.11). As already stated in Remark 6.3 this is equivalent to our triple splitting method (3.1) with $\psi_{B} \equiv \phi_{B} \equiv 1$. The two-step formulation with the distinct first step is equivalent to [6, Scheme (3)] with the filter functions $\phi$ and $\psi$ as in (6.13), $\psi(z)=\operatorname{sinc}(z) \psi_{1}(z)$, 
and $\psi_{0}(z)=\cos (z) \psi(z)$. For a second-order error estimate for the scheme (3.1) with $\psi_{B} \equiv \phi_{B} \equiv 1$, we then require (5.2d)-(5.2h) as before but replace the first three assumptions (5.2a)-(5.2c) by

$$
\begin{aligned}
\left|1-\phi_{E}\left(\frac{1}{2} z\right)\right| & \leq C_{9}|z| \\
\left.\mid \operatorname{sinc}^{2}\left(\frac{1}{2} z\right)-\frac{1}{2}(\cos (z)+1) \psi_{E}\left(\frac{1}{2} z\right)\right) \mid & \leq C_{10}\left|\sin \left(\frac{1}{2} z\right)\right| \\
\left|\operatorname{sinc}(z)-\phi_{E}\left(\frac{1}{2} z\right)\right| & \leq C_{11}\left|z \sin \left(\frac{1}{2} z\right)\right| \\
\left|\operatorname{sinc}^{2}(z)-\frac{1}{2}(\cos (z)+1) \psi_{E}\left(\frac{1}{2} z\right)\right| & \leq C_{12}\left|\sin (z) \sin \left(\frac{1}{2} z\right)\right| \\
\left|\operatorname{sinc}^{2}(z)-\frac{1}{2}(\cos (z)+1) \psi_{E}\left(\frac{1}{2} z\right) \cos (z)\right| & \leq C_{13}\left|\sin (z) \sin \left(\frac{1}{2} z\right)\right| .
\end{aligned}
$$

Assumptions (5.2d) and (5.2g) yield

$$
|\eta(z)| \leq \max \left\{2 C_{4}, C_{7}\right\}
$$

for $\eta \in\left\{\phi, \psi, \psi_{0}, \psi_{1}\right\}$, which is [6, Condition (11)]. The new assumption (7.1a) yields

$$
|(\phi(z)-1)| \leq C_{9}|z|,
$$

which is [6, Condition (12)]. (7.1b) yields

$$
\left|\left(\operatorname{sinc}^{2}\left(\frac{1}{2} z\right)-\psi(z)\right)\right| \leq C_{10}\left|\sin \left(\frac{1}{2} z\right)\right|,
$$

which is [6, Condition (13)]. The filter assumptions (7.1c), (7.1d), and (7.1e) yield

$$
|(\operatorname{sinc}(z)-\chi(z))| \leq C_{13}\left|z \sin \left(\frac{1}{2} z\right)\right|
$$

for $\chi=\phi, \psi_{0}, \psi_{1}$, which is [6, Condition (14)]. The conditions [6, Condition (11)-(14)] are sufficient for a second-order estimate of the solution (without the derivative), cf. [6, Theorem 1], which is all we need.

Our proposed filter choice (5.4) in addition to the filter conditions (5.2) also fulfills the new filter conditions (7.1); (7.1d) holds true with $C_{12}=0$. This implies that scheme (3.1) with $\psi_{B} \equiv \phi_{B} \equiv 1$ and (5.4) is of second order also for multiple high frequencies in $\Omega$.

\section{Numerical experiments.}

8.1. Laser plasma interaction-triple splitting. To illustrate the convergence, we carry out an experiment similar to the one presented in [17].

The setting is taken from the thin foil experiment in Section 2. We use the laser pulse from (2.3) as initial value for the fields and a zero initial impulse. In vacuum, this models a laser pulse propagating in positive $x$-direction. We assume that the domain is homogeneous in $y$ - and $z$-directions such that the continuous equations (2.1) decouple, and it is sufficient to consider the simplified equations

$$
\begin{aligned}
\partial_{t} \mathbf{p}_{y} & =\mathbf{e}_{y}, \\
\partial_{t} \mathbf{e}_{y} & =-\partial_{x} \mathbf{b}_{z}-f^{2} \rho \mathbf{p}_{y}, \\
\partial_{t} \mathbf{b}_{z} & =-\partial_{x} \mathbf{e}_{y},
\end{aligned}
$$

where only the $y$ - and $z$-components of the fields are taken into account. Note that these are functions of $t$ and $x$ only. For simplicity, periodic boundary conditions in $x$ are used. The density profile is chosen as

$$
\rho(x)= \begin{cases}\rho_{F}, & \text { if } x \in F, \\ 0, & \text { otherwise }\end{cases}
$$


where $F=[20,21]$ is the area covered by the foil. Except for $\rho_{F}$, which will be varied, all other physical parameters are chosen as in Section 2, i.e., material parameters, wavelength, etc. The spatial discretization of the computational domain $B=[0,24]$ is done with central finite differences for the spatial derivative of the magnetic flux $\mathbf{b}$ and the electric field $\mathbf{e}$ on a staggered grid with 241 equispaced grid points. This corresponds to the staggered Yee grid translated to the one-dimensional situation. The conditions from Assumption 4.1 on the discretization are thus satisfied. The bounds for the initial data from Assumption 4.2 are also satisfied, exploiting that $|\mathbf{e}|$ and $|\mathbf{b}|$ are smaller than machine precision in the region of the foil $F$, and thus the error from setting them to zero is not larger then the round-off error when evaluating the exponential function numerically.

In Figure 8.1 we present the error in $\mathbf{e}, \mathbf{p}$, and $\mathbf{b}$. The error in $\mathbf{e}$ dominates the error in $\mathbf{b}$ by almost one magnitude. The error in the impulse $\mathbf{p}$ almost coincides with the error in the electric field $\mathbf{e}$ if no filters are used, and it is thus not visible in the plots. If the filter choice (5.3) is employed, then the error in $\mathbf{e}$ and $\mathbf{p}$ coincide away from even multiples of $\pi$. In the inset of the second panel in the second row, one can see that the error in $\mathbf{p}$ does not "peak". Thus the peaks in this case are in the error of e only. The left column in Figure 8.1 displays the error of the method for $\rho_{F}=64 \cdot 10^{8}, \omega=8 \cdot 10^{4}$, and the right column corresponds to a plasma with $\rho_{F}=9 \cdot 10^{6}, \omega=3 \cdot 10^{3}$. We display the Euclidean norm of the absolute error at $T=20$ versus the step size $\tau$ for the numerical solution of (3.1) measured against the spatially discrete reference solution (2.2) calculated with the expmv routine from [1]. In the upper row, no filter functions were used resulting in large broad error peaks. In the middle row, the filter choice (5.3) results in very sharp error peaks around even multiples of $2 \pi / \omega$. As predicted by our theory, the bottom row shows a second-order convergence independent of $\omega$. For the zoom, the range of step sizes is $\tau \in[0.923 \cdot 2 \pi / \omega, 1.075 \cdot 2 \pi / \omega]$ if no filter function is used, and it is much smaller if a filter function is chosen, i.e, $\tau \in[0.997 \cdot 2 \pi / \omega, 1.003 \cdot 2 \pi / \omega]$.

8.2. Klein-Gordon-type equation-two-step method. To illustrate the results and remarks from Section 6.2 we consider a one-dimensional Klein-Gordon-type equation for one component of the electric field with periodic boundary conditions on the interval $[-10,14]$, where the plasma occupies the region $(10,11)$. This equation is obtained by eliminating $\mathbf{b}$ and $\mathbf{p}$ from (2.1). Discretization in space is achieved by symmetric second-order finite differences on the equidistant grid $x$ with grid points $x_{j}=-10+j h, j=0, \ldots, N$, with $N=240$ and a spacing of $h=24 / N$. The initial value $\mathbf{e}_{0}$ is given by (2.3) ( $\left.\bar{x}=0, \sigma_{0}=10\right)$ evaluated on the grid and the initial velocity by $\left(\dot{\mathbf{e}}_{0}\right)_{j}=\left(\left(\frac{2 \pi}{\sigma_{0}}\right)^{2} x_{j} \cos \left(2 \pi x_{j}\right)+2 \pi \sin \left(2 \pi x_{j}\right)\right) \exp \left(-\frac{2 \pi^{2}}{\sigma_{0}^{2}} x_{j}^{2}\right)$. The equation that we solve for $\mathbf{e}(t)$ is

$$
\begin{aligned}
\partial_{t t} \mathbf{e}(t) & =\mathbf{G e}(t)-\mathbf{\Omega} \mathbf{e}(t), \quad \text { for } t \in[0,20], \\
\mathbf{e}(0) & =\mathbf{e}_{0}, \quad \partial_{t} \mathbf{e}(0)=\dot{\mathbf{e}}_{0},
\end{aligned}
$$

with, using Matlab notation, the matrices

$$
\mathbf{G}=\operatorname{spdiags}([e,-2 * e, e],-1: 1, N, N) / h^{2}, \quad \mathbf{G}(1, N)=1 / h^{2}, \quad \mathbf{G}(N, 1)=1 / h^{2},
$$

for a vector $e$ with all ones and $\boldsymbol{\Omega}=\operatorname{diag}(\omega * f)$, where the diagonal $f$ is represented by a step function, i.e., $f=z \operatorname{eros}(\operatorname{size}(x)) ; f(x<11 \& x>10)=1$ for a given $\omega$.

We have implemented the two-step method from [8, XIII.2.2] with even real-valued filter 
functions $\psi$ and $\phi$, with $\psi(0)=\phi(0)=1$.

$$
\begin{array}{ll}
\text { (A) } & \psi(z)=\operatorname{sinc}^{2}\left(\frac{1}{2} z\right) \\
\text { (B) } & \psi(z)=\operatorname{sinc}(z) \\
(\mathrm{C}) & \psi(z)=\operatorname{sinc}\left(\frac{1}{2} z\right) \phi(z) \\
(\mathrm{D}) & \psi(z)=\operatorname{sinc}^{2}\left(\frac{1}{2} z\right) \\
(\mathrm{E}) & \psi(z)=\operatorname{sinc}^{2}(z) \\
(\mathrm{F}) & \psi(z)=\eta(z) \operatorname{sinc}\left(\frac{1}{2} z\right)^{2} \\
(\mathrm{G}) & \psi(z)=\eta(z) \operatorname{sinc}\left(\frac{1}{2} z\right) \\
(\mathrm{H}) & \psi(z)=\operatorname{sinc}\left(\frac{1}{2} z\right) \\
(\mathrm{I}) & \psi(z)=\operatorname{sinc}(z)
\end{array}
$$$$
\phi(z)=1
$$$$
\phi(z)=1
$$$$
\phi(z)=\operatorname{sinc}(z)
$$$$
\phi(z) \text { from (8.3) below }
$$$$
\phi(z)=1
$$$$
\phi(z)=\operatorname{sinc}\left(\frac{1}{2} z\right)
$$$$
\phi(z)=\operatorname{sinc}\left(\frac{1}{2} z\right)
$$$$
\phi(z)=\operatorname{sinc}(z)
$$

where

$$
\phi(z)=\operatorname{sinc}(z)\left(1+\frac{1}{3} \sin ^{2}\left(\frac{1}{2} z\right)\right)
$$

in the method (D). The alphabetic labels for the methods (A)-(E) follow the convention of [8]. Method (F) corresponds to our choice, (5.4), with the "natural filter" $\eta(z):=\frac{1}{2}(1+\cos (z))$ coming for free from the triple splitting. Method (G) corresponds to the choice (5.3) considered in $[13,17]$.

Figure 8.2 displays the absolute error in the Euclidean norm versus the time step size. For this linear test problem, method (E) shows the same behavior as (A), the behavior of (D) is similar to (C), and (H) is similar to (I). Therefore the results for (E), (D), and (H) are not displayed. The inset is a zoom to step sizes around $2 \pi / \omega$ giving the error for $\tau$ in the interval $\tau \in\left[\left(2 \pi-5 \cdot 10^{-3}\right) / \omega,\left(2 \pi+5 \cdot 10^{-3}\right) / \omega\right]$. For this linear test problem one observes a second-order convergence as soon as there is a double zero of $\psi$ at even multiples of $\pi$. The condition on $\phi$ seems to be less important. However comparing (I) and the method (B), it can be observed that the resonance peak is much sharper for (I), reflecting the influence of $\phi$ in this test problem. Though the filter functions of the methods (G), (H), and (I) satisfy the assumptions for first-order convergence uniformly in $h \omega$ as predicted by [8, Theorem XIII.4.1], cf. Remark 6.2, sharp resonance peaks are observed. Hence, this experiment is a counterexample to the claim made there. The second-order convergence result with the more restrictive assumptions on the filter functions remains valid.

Acknowledgments. We thank Ernst Hairer and Ludwig Gauckler for answering all our questions when we were carefully checking the proof of Theorem 6.1 and the relevant part of [8, Theorem XIII.4.1]. Ernst Hairer helped to close a gap in the proof. We thank Marlis Hochbruck and Volker Grimm for their interest in our work and stimulating discussions.

\section{REFERENCES}

[1] A. H. Al-Mohy And N. J. Higham, Computing the action of the matrix exponential, with an application to exponential integrators, SIAM J. Sci. Comput., 33 (2011), pp. 488-511.

[2] S. BUCHHOLZ AND M. HoCHBRUCK, Error analysis of hybrid particle-in-cell (PIC) methods for oscillatory Maxwell-like equations, in Book of Abstracts of the 12th Int. Conf. on Math. and Numer. Aspects of Wave Propagation, KIT, Karlsruhe, 2015.

[3] P. Deuflhard, A study of extrapolation methods based on multistep schemes without parasitic solutions, Z. Angew. Math. Phys., 30 (1979), pp. 177-189.

[4] B. García Archilla, J. M. SanZ-Serna, And R. D. SkeEL, Long-time-step methods for oscillatory differential equations, SIAM J. Sci. Comput., 20 (1999), pp. 930-963. 
[5] W. GAUTSCHI, Numerical integration of ordinary differential equations based on trigonometric polynomials, Numer. Math., 3 (1961), pp. 381-397.

[6] V. GRIMM AND M. HOCHBRUCK, Error analysis of exponential integrators for oscillatory second-order differential equations, J. Phys. A, 39 (2006), pp. 5495-5507.

[7] E. HAIRER AND C. LUBICH, Long-time energy conservation of numerical methods for oscillatory differential equations, SIAM J. Numer. Anal., 38 (2000), pp. 414-441.

[8] E. Hairer, C. Lubich, AND G. WAnner, Geometric Numerical Integration, 2nd ed., Springer, Berlin, 2006.

[9] R. HiptMAIR, Finite elements in computational electromagnetism, Acta Numer., 11 (2002), pp. 237-339.

[10] R. Hiptmair, P. R. Kotiuga, And S. Tordeux, Self-adjoint curl operators, Ann. Mat. Pura Appl. (4), 191 (2012), pp. 431-457.

[11] M. HOCHBRUCK AND C. LUBICH, A Gautschi-type method for oscillatory second-order differential equations, Numer. Math., 83 (1999), pp. 403-426.

[12] G. JANSING, Exponentielle Integratoren-Zeitintegrationsverfahren für Maxwell-Gleichungen und Parabolische Systeme, PhD. Thesis, Math.-Nat. Fakultät, Heinrich-Heine Universität Düsseldorf, Düsseldorf, 2015.

[13] J. LiLjo, Hybride Verfahren zur Simulation der Wechselwirkung Relativistischer Kurzpuls-Laser mit Hochdichten Plasmen, PhD. Thesis, Math.-Nat. Fakultät, Heinrich-Heine Universität Düsseldorf, Düsseldorf, 2010.

[14] J. Liljo, A. Karmakar, A. Pukhov, And M. Hochbruck, One-dimensional electromagnetic relativistic PIC-hydrodynamic hybrid simulation code H-VLPL (hybrid virtual laser plasma lab), Comput. Phys. Comm., 179 (2008), pp. 371-379.

[15] T. Tückmantel, Hybrid Particle-in-Cell Simulations of Relativistic Plasmas, PhD. Thesis, Math.-Nat. Fakultät, Heinrich-Heine Universität Düsseldorf, Düsseldorf, 2013.

[16] T. TÜCKMANTEl AND A. PUKhOv, H-VLPL: a three-dimensional relativistic PIC/fluid hybrid code, J. Comput. Phys., 269 (2014), pp. 168-180.

[17] T. TÜCKMAntel, A. Pukhov, J. Liljo, AND M. Hochbruck, Three-dimensional relativistic particle-incell hybrid code based on an exponential integrator, IEEE Trans. Plasma Sci., 38 (2010), pp. 2383-2389. 

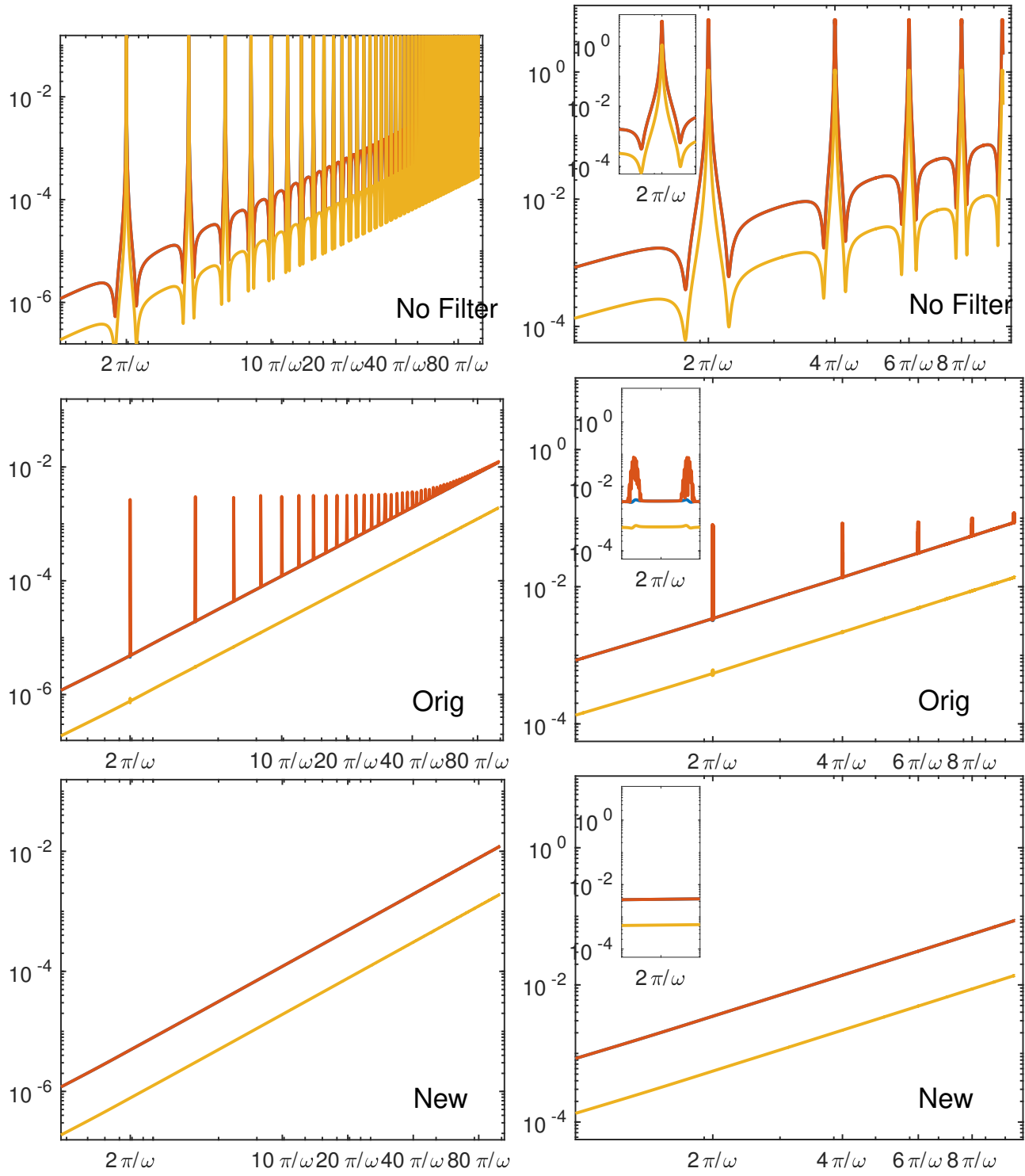

FIG. 8.1. Euclidean norm of the absolute error of $\mathbf{e}, \mathbf{p}$, and $\mathbf{b}$ at $T=20$ versus the step size for the numerical solution (3.1) measured against the spatially discrete reference solution of (2.2). Left: $\omega=8 \cdot 10^{4} ;$ right: $\omega=3 \cdot 10^{3}$; top: no filter; middle: filter of [17] (Orig); bottom: new filter choice (5.4) (New). 



FIG. 8.2. Euclidean norm of the absolute error at $T=3$ versus the step size for the Klein-Gordon-type equation (8.2). Results are shown for the filter choices $(A),(B),(C),(G),(I)$, and $(F)$. The inset shows a zoom to step sizes in the interval $\left[\left(2 \pi-5 \cdot 10^{-3}\right) / \omega,\left(2 \pi+5 \cdot 10^{-3}\right) / \omega\right]$. 